\title{
Space Weathering of S-Complex Asteroids Manifested in the UV/Blue: Recent Insights and Future Directions
}

\author{
Faith Vilas ${ }^{1}$, and Amanda R. Hendrix ${ }^{2}$ \\ ${ }^{1}$ Planetary Science Institute \\ 1700 E. Fort Lowell Rd., Suite 106 \\ Tucson, AZ 85719 USA \\ email: fvilas@psi.edu \\ ${ }^{2}$ Planetary Science Institute \\ 1700 E. Fort Lowell Rd., Suite 106 \\ Tucson, AZ 85719 USA \\ email: ahendrix@psi.edu
}

\begin{abstract}
Space weathering affects reflectance spectra of the Moon and S-complex asteroids by spectral bluing (increasing reflectance with decreasing wavelength) of their surface materials at UV/blue (less than $400 \mathrm{~nm}$ ) wavelengths. This spectral bluing is attributed to a degradation of the UV absorption feature seen in spectral reflectance of olivine as a result of the creation of nanophase $\left(\mathrm{npFe}^{0}\right)$ iron. We have modeled the effect of the addition of small amounts of $\mathrm{npFe}^{0}$ intimately mixed with particles from a hypothetical material and a terrestrial basalt. The addition of $0.0001 \% \mathrm{npFe}^{0}$ affects the reflectance at these UV/blue wavelengths, while the addition of $0.01 \%$ is required to see the visible/near infrared reddening and diminution of VNIR absorption features. Thus, the UV/blue spectral reflectance characteristics allow earlier detection of the onset of space weathering effects.
\end{abstract}

Keywords. minor planets, asteroids; planets and satellites: general; ultraviolet: solar system

\section{Introduction}

Over 690,000 asteroids have been discovered and numbered by October, $2015^{1}$. Despite sample return missions to near-Earth asteroids (NEAs) both in progress and successfully completed, remote telescopic sensing remains the primary means we use to study these objects. The phenomenon of "space weathering" refers to the alteration of properties of surface materials by processes active in the space environment (e.g., heavy ion bombardment, micrometeoroid bombardment): all atmosphereless bodies are subject to different forms of space weathering. Thus, accurate interpretation of telescopic data requires proper consideration of any space weathering effects on the surface material.

Pristine samples of the Moon were retrieved as part of the Apollo and Luna programs. The Hayabusa mission to S-complex asteroid 25143 Itokawa retrieved pristine samples of an NEA (e.g., Nakamura et al. 2011). From both of these sources, it is apparent that the space weathering affecting these objects is due to grain coatings and inclusions of nanophase iron $\left(\mathrm{npFe}^{0}\right)$ on olivine present in the surface regolith of the Moon, and grain coatings containing nanophase $(\mathrm{Fe}, \mathrm{Mg}) \mathrm{S}$ coupled with partially amorphized olivine and low-Ca pyroxene containing nanophase $\mathrm{Fe}\left(\mathrm{npFe}^{0}\right)$ on small Itokawa particles (e.g.,

\footnotetext{
According to Horizons (http://ssd.jpl.nasa.gov/?horizons), the Jet Propulsion Laboratory online ephemeris system.
} 
Pieters et al. 1993; Noble et al. 2001; Noguchi et al. 2011, 2014). In the visible/nearinfrared (VNIR, > $600 \mathrm{~nm}$ ) spectral region, this space weathering darkens and reddens (increasing reflectance with increasing wavelength) the reflectance spectra of the objects, as well as degrading any spectral absorption features (e.g., Chapman 1996). This is the canonical image of space weathering, first identified with lunar samples. Laboratory reflectance spectra of lunar soils retrieved from the surface and containing the $n p F e^{0}$ show these spectral effects, while laboratory reflectance spectra of freshly-ground fine lunar samples containing no $\mathrm{npFe}^{0}$ do not show them (e.g., Pieters et al. 1993). The spectral effects of the presence of the $\mathrm{npFe}^{0}$ in lunar laboratory samples are echoed in remotely-sensed reflectance spectra of areas across the lunar surface.

Spectral changes across the Moon first led to investigations of the effects of $n p F e^{0}$ creation in the ultraviolet. Lunar maria are observed to be darker than lunar highlands in the VNIR. Observations of the moon in the extreme ultraviolet, however, show that lunar maria are 5 - $10 \%$ brighter than the lunar highlands at far-UV wavelengths (147 nm) (Lucke et al. 1974; Flynn et al. 1998). Laboratory reflectance spectra of weathered lunar soils also show this spectral reversal in the EUV, while those of ground lunar samples do not, pointing to a connection of this spectral reversal with the $\mathrm{nFFe}^{0}$ created as part of the space weathering (Wagner et al. 1987).

At VNIR wavelengths, particulate spectral reflectance characteristics are governed by volume scattering (e.g., Hapke 2001), which includes a grain-size dependency to the intensity as

$$
\text { Intensity } \sim \exp (-\alpha D), \quad \alpha=4 \pi k / \lambda,
$$

where $\mathrm{D}$ is grain diameter. This is supported by the observation that space weathering effects at VNIR wavelengths are detected primarily in fine regoliths (e.g., Pieters et al. 1993). At UV/blue wavelengths, particulate spectral reflectance characteristics are governed by surface scattering controlled by Fresnel reflection, which contains no dependency on grain size:

Fresnel reflection coefficient $R \sim\left[(n-1)^{2}+k^{2}\right] /\left[(n+1)^{2}+k^{2}\right]$,

where $\mathrm{n}, \mathrm{k}$ are the optical constants of the material. For non-opaque materials such as mafic silicates, the transition from surface to volume scattering occurs within the 150to-450-nm wavelength range (Wagner et al. 1987). Fresnel reflection dominates opaque materials across all spectral regions, resulting in spectrally "flat" reflectance.

\section{Evidence for Space Weathering in the UV/Blue on S-Complex Asteroids}

Recent, extensive laboratory studies show that the creation of $\mathrm{npFe}^{0}$ is due to heavy ion irradiation on both particulates and fragments of Fe-bearing olivines, and that this irradiation causes space weathering effects over twice as rapidly as micrometeoroid bombardment at the distance of 1 AU (Loeffler et al. 2009). We investigate a scenario for S-complex asteroid beginning with the presence of fresh olivine on the asteroid's surface, in grains of varying size (Vilas \& Hendrix 2015a). As soon as a surface is exposed directly to space, an $\mathrm{nFe}^{0}$ coating and inclusions will begin to form on the fresh surface. Without the requirement for small particle sizes, the bluing (increasing reflectance with decreasing wavelength) of the surface reflectance spectrum in the UV/blue spectral region starts immediately as the $\mathrm{nFFe}^{0}$ coating begins to mask the olivine and the UV absorption diminishes. With time, more $\mathrm{nFe}^{0}$ will be added as more weathering occurs, coupled with likely micrometeoroid bombardment breaking particles into smaller sizes. 
At this stage, typical lunar-like space weathering effects will now be seen in the VNIR reflectance spectra of the asteroid.

We have proposed and tested the possibility that the UV/blue range could be a more sensitive detector of space weathering. Ultraviolet spectra acquired with IUE (185 - 335 $\mathrm{nm})$, HST (222 - $326 \mathrm{~nm})$, and Galileo (162 - $323 \mathrm{~nm}$ ) coupled with ground-based VNIR spectra of S-complex asteroids, and compared with spectra of H, L, and LL ordinary chondrite meteorites, showed similar differences between S-complex asteroids exposed to the space environment and pristine ordinary chondrite meteorites (Hendrix \& Vilas 2006). We further defined the spectral interval of 320 - $400 \mathrm{~nm}$ as most effective for observing the UV/blue effects of the presence or absence of space weathering from groundbased telescopes (Vilas \& Hendrix 2015a). Using ground-based high-resolution UV/blue telescopic spectra of asteroids observed by spacecraft in the UV and selected near-Earth, main-belt, and Mars crossing asteroids, we demonstrate that a downward turn or "kink" is present in the spectra of the S-complex asteroids near $370-390 \mathrm{~nm}$. This downturn is visually apparent in some NEA and young-family main-belt asteroids where we expect comparatively less $\mathrm{npFe}{ }^{0}$ creation, but diminished in mature S-complex asteroid spectra (Vilas \& Hendrix 2015a). We propose that we observe the visual manifestation of this space weathering process in the UV/blue. We modeled intimate mixtures of particles from a hypothetical material and from a terrestrial basalt (as an analog for olivine, due to the lack of with increasing amounts of $n \mathrm{pFe}^{0}$. We then demonstrate that the onset of space weathering becomes apparent in a UV/blue spectrum at wavelengths below $400 \mathrm{~nm}$ with the addition of $0.0001 \% \mathrm{npFe}^{0}$ to an S-complex surface, and becomes apparent in the VNIR with the addition of $0.01 \% \mathrm{npFe}^{0}$ (Vilas \& Hendrix 2015a). Thus, we believe that the $\mathrm{UV} /$ blue spectral region is a more sensitive indicator of the onset of space weathering on a fresh surface. Figure 1 demonstrates the relationship between the VNIR and NUV spectral slopes, and delineates the regions containing varying amounts of $n p F e^{0}$.

\section{Future Directions}

This knowledge allows scientists to probe different aspects of the space weathering of S-complex asteroids, or indeed any exposed olivine surface in the solar system. We consider here two possibilities that could advance our understanding of the effects on olivine-bearing surfaces exposed in the solar system, and concomitantly the onset and presence of this type of space weathering among the S-complex asteroids. We identify some questions to be studied here in the future:

\subsection{Evidence in Broad Band Photometry}

In Vilas et al. (2015b), the ability to sample S-complex surfaces for the presence and level of space weathering as evidenced in the creation of $\mathrm{npFe}^{0}$ on olivine samples was shown to persist across the 360-to-440 nm break present between the U and B (or, u' and g' Sloan Digital Sky Survey) broad band filters. This allowed SDSS observations of small main-belt asteroids to be used to identify candidates for future observations. What do higher resolution UV/blue and VNIR reflectance spectra of these asteroids tell us about the creation of $\mathrm{nFe}^{0}$ in specifically the main asteroid belt?

\subsection{Differences in the UV Spectral Properties}

Laboratory studies of the grain coatings and rims observed in lunar fines retrieved by the Apollo missions, as well as microscopic particles retrieved from 25143 Itokawa by the Hayabusa mission, show differences in the physical structure within the first $100 \mathrm{~nm}$ of the weathering coatings on the surfaces of these particles (e.g., Thompson et al. 2014; 


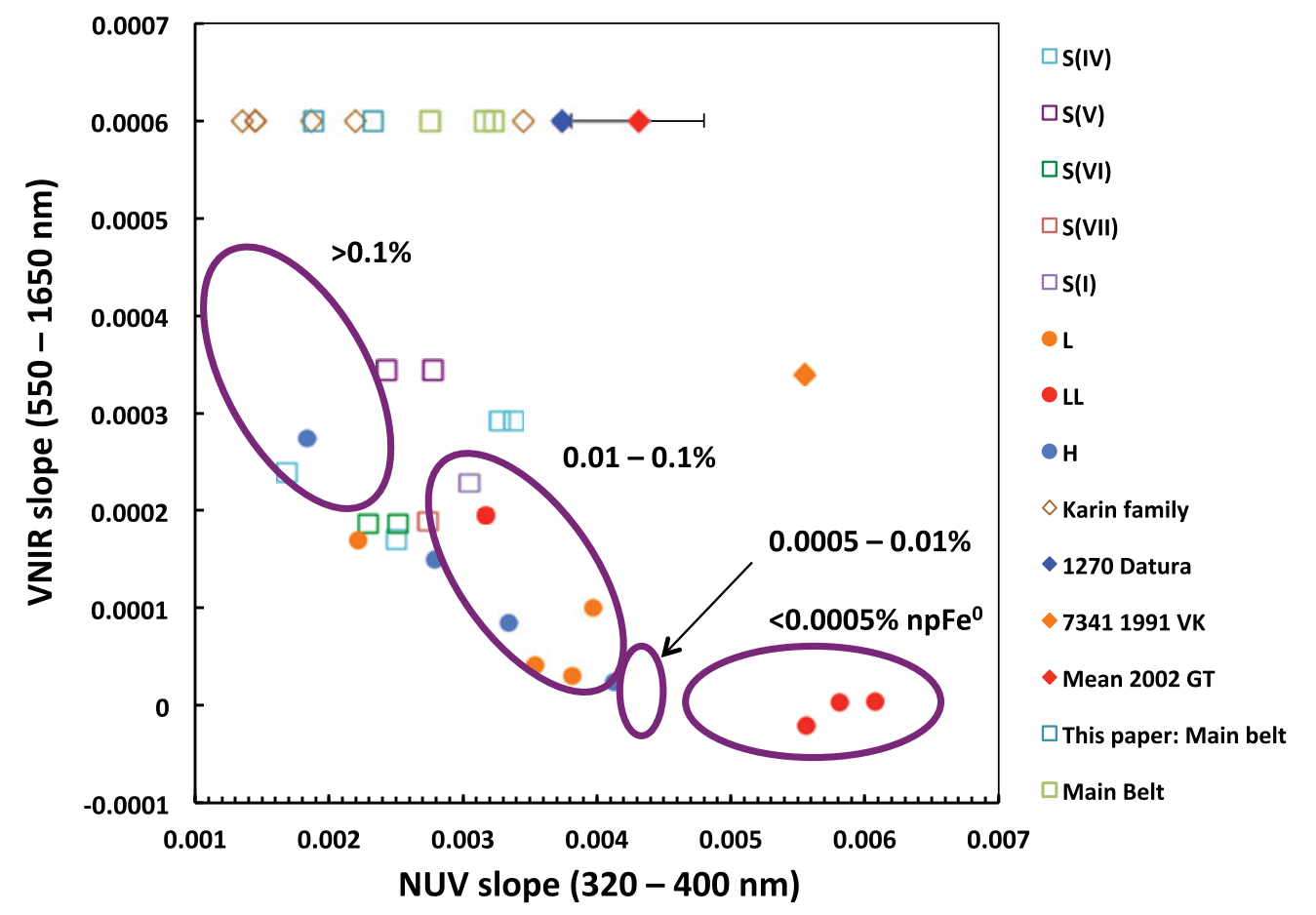

Figure 1. Spectral slopes (in units of reflectance $\mathrm{nm}^{-1}$ ) of S-complex asteroids and ordinary chondrite meteorites. Where VNIR slopes are not available, spectra have been plotted with VNIR slope $=0.0006 \mathrm{~nm}^{-} 1$. For asteroid 163249, an average of 13 observations with error is plotted. The mean 1- $\sigma$ error for the newly calculated UV slopes of the CCD reflectance spectra is plotted on the $\mathrm{S}(\mathrm{I})$ asteroid open square. The mean 1- $\sigma$ errors for the UV slopes of laboratory measurements of ordinary chondrite meteorites is contained within the symbols for the meteorite slopes. The ovals mark regions indicating modeled percentages of $\mathrm{npFe}^{0}$ intimately mixed with a terrestrial basalt as an analog for olivine. These correlate with the observed UV/blue slopes. Coupled with distance from the sun, this information can be used to date S-complex and Q- and O-type asteroids that are in the early stages of space weathering. Figure adapted from Vilas \& Hendrix (2015a).

Keller \& Berger 2014). These structural differences support solar wind heavy ion irradiation and bombardment as most likely to affect the surface properties of the S-complex asteroids, and micrometeoroid bombardment as the source of weathering on the lunar surface. The surface scattering region in the UV does not penetrate deeply, and so is sensitive to these thin, nanophase outer layers. Can we use UV spectra to distinguish among structural characteristics of space weathering products to determine the weathering creation circumstances?

There is always a need for additional spectral data across the UV - VIS - NIR wavelength range. Despite concerted efforts to acquire NIR spectra of NEAs in recent years, most young family asteroids and NEAs observed in our UV/blue program have not been observed in the NIR. More observations fill in the relationship shown in Fig. 1. The EUV spectra of the Moon extend to shorter wavelengths than the telescopic UV spectra of S-complex asteroids. Would EUV spectra of S-complex asteroids also show the spectral reversal seen in the lunar EUV data? The apparent magnitudes of these objects at the Earth's distance preclude these observations unless they are acquired in close proximity to the asteroid. Future remote telescopic observations rely on these current studies to define and quantify the effects of space weathering. 


\section{Acknowledgments}

This is a SSERVI Center for Lunar and Asteroid Surface Science publication.

\section{References}

Chapman, C. R. 1996, Meteorit. Planet. Sci., 31, 699

Flynn, B. C., Vallerga, J. V., Gladstone, G. R., \& Edelstein, J. 1998, Geophys. Res. Lett., 25, 3253

Hapke, B. 2001, J. Geophys. Res., 106, 10039

Hendrix, A. R. \& Vilas, F. 2006, Astron. J., 132, 1396

Keller, L. P. \& Berger, E. L. 2014, LPI Contributions, 1800, 5088

Loeffler, M. J., Dukes, C. A., \& Baragiola, R. A. 2009, J. Geophys. Res., 114, E03003

Lucke, R. L., Henry, R. C., \& Fastie, W. G. 1974, Lunar and Planetary Science Conference, 5, 469

Nakamura, T., Noguchi, T., Tsuchiyama, A., et al. 2011, AGU Fall Meeting Abstracts, A2

Noble, S. K., Pieters, C. M., Taylor, L. A., et al. 2001, Meteorit. Planet. Sci., 36, 31

Noguchi, T., Nakamura, T., Kimura, M., et al. 2011, Science, 333, 1121

Noguchi, T., Kimura, M., Hashimoto, T., et al. 2014, Meteorit. Planet. Sci., 49, 188

Pieters, C. M., Fischer, E. M., Rode, O., \& Basu, A. 1993, J. Geophys. Res., 98, 20817

Thompson, M. S., Christoffersen, R., Zega, T. J., \& Keller, L. P. 2014, Earth, Planets, and Space, 66, 89

Vilas, F. \& Hendrix, A. R. 2015a, Astron. J., 150, 64

Vilas, F., Hendrix, A. R., \& Jensen, E. A. 2015b, Planet. Space Sci., in press

Wagner, J. K., Hapke, B. W., \& Wells, E. N. 1987, Icarus, 69, 14 